\title{
Chromosomal abnormalities in a decade of prenatal testing at the Department of Obstetrics and Pathology of Pregnancy Medical University of Lublin
}

\begin{abstract}
Introduction. Chromosomal abnormalities, one of the leading causes of pregnancy complications, attract attention of both researchers and clinicians. They use two approaches to identify chromosomal abnormalities, namely screening and diagnostic tests. Ultrasonography is a very reliable screening and diagnostic tool, but the only way to determine if there are any chromosomal defects in the fetus, is performing one of invasive diagnostics tests chorionic villus sampling (CVS), cordocentesis or amniocentesis. Unfortunately, these invasive diagnostic procedures carry a potentially high risk of complications. Using amniocentesis means a procedure-related miscarriage risk at a rate of about $0.5-1 \%$.

Aim. The aim of this paper was to present our own experience, results in performing amniocentesis and a review of the literature.

Material and methods. During a 10-year period 237 mid-trimester, transabdominal amniocenteses were performed.

Results. The follow-up revealed one spontaneous abortion within seven days after the procedure. Premature delivery occurred in fourteen cases (two of them with chromosomal abnormalities). No neonatal deaths related to amniocentesis were noticed. Chromosomal abnormalities were detected in 33 patients.

Conclusions. In the group with chromosomal abnormalities the main indications to perform amniocentesis were: improper ultrasound scan and the first trimester biochemical, noninvasive screening tests. This is a proof that modern, non-invasive procedures like the first-trimester ultrasound scan and biochemical tests should be made available to every pregnant woman and not only to mothers' aged $>35$ years or those with a poor obstetrics history.
\end{abstract}

Keywords: pregnancy, amniocentesis, screening test, Down Syndrome, chromosomes.

DOI: $10.1515 /$ pjph-2015-0018

\section{INTRODUCTION}

Prenatal screening is an important public health strategy that counters common genetic disorders. Prenatal screening for fetal chromosomal abnormalities is a rapidly evolving science and an essential part of antenatal care because chromosomal defects are still the leading cause of pregnancy complications. Nowadays, an increasing number of fetal abnormalities can be detected due to the variety of advanced prenatal diagnostic techniques available. It is well known that the risk for chromosomal defects like trisomy 21 is directly related to maternal age. And even though only about 30 percent of infants with Down syndrome are born to these women [1]. Despite that, if a mother's age exceeds 35 years, there is a strict need for invasive diagnostic tests. A variety of screening and diagnostic tests can be applied to assess the risk and detect chromosomal abnormalities [2,3]. Current diagnostic methods during the first trimester include: detailed ultrasonography performed between 11-13+6 weeks of gestation integrated with some maternal serum sampling (measurement of pregnancy-associated plasma protein A (PAPPA) and free human chorionic gonadotropin (B-HCG) levels. Ultrasound markers of fetal congenital abnormalities or genetic syndromes found during the first trimester scanning include: increased nuchal transcluency (NT), absence of the nasal bone (NB), hypoplastic maxilla, FMF - Fronto Maxiliary Facial angle, abnormal blood flow velocity in the fetal ductus venosus (DV), Tricuspid Valve flow, single umbilical artery and omphalocele. The combination of NT, NB, DV, TV flow with the first-trimester biochemical markers is more effective than either alone. The detection rate of a combined test is about $85-90 \%$ in regard to trisomy 21 and 18 , with a false positive rate of 5\%. Many authors recommend combined testing for first-trimester screening [4-6]. Screening options in the second trimester include triple or quadruple screening tests, ultrasonography performed in 18-24 hbd and prenatal echocardiography. The "triple test", which is performed usually between 15 and 17 weeks gestation, includes HCG, alpha-fetoprotein (AFP) and unconjugated estriol (E3) concentrations. The "quadruple" test is a combination of triple screen with inhibin A. Second trimester ultrasound markers of fetal congenital abnormalities or genetic syndromes include: congenital heart defects, hyperechogenic intracardiac foci, holoprosencephaly, agenesis 
of corpus callosum, cleft lip, micrognathia, encephalocele, omphalocele, micrognathia, cystic hygroma.

Screening tests are helpful, but the only way to determine chromosomal abnormality, is to perform an invasive test: chorionic villus sampling (CVS), cordocentesis or amniocentesis [7-10]. These procedures involve direct examination of fetal cells or tissues. The diagnostic prenatal tests were introduced into clinical practice in the 1970s. One of the above-mentioned invasive procedures should be offered to both women aged at least 35 years and those with unsatisfactory prenatal screening tests results. When considering invasive techniques, all indications must be carefully evaluated as these procedure's may cause complications and increase the risk of pregnancy loss.

The discovery of cell-free fetal DNA (cffDNA) in maternal blood offered new opportunities for noninvasive prenatal diagnosis [11]. This technology offered an easier and safer method of prenatal testing than CVS, amniocentesis or cordocentesis. It is likely to become widely used in the future.

\section{AIM}

The aim of this paper was to present our own experience and consideration in performing amniocentesis. We analyzed the indications, results and complications of this procedure and discussed advantages and disadvantages of invasive tests.

\section{MATERIAL AND METHODS}

During a 10-year period (2005-2014), 237 mid-trimester transabdominal amniocenteses between 16 and 23 weeks of gestation were performed. Maternal age ranged from 16 to 47 years old. The gestational age at the day of procedure was: 16 weeks in $3 \%(7 / 237), 17$ weeks in $21.9 \%(52 / 237)$, 18 weeks in $37.2 \%(88 / 237), 19$ weeks in $18.6 \%(44 / 237)$, 20 weeks in $10.6 \%(25 / 237), 21$ weeks in $4.3 \%(10 / 237), 22$ weeks in $3 \%(7 / 237), 23$ weeks in $0.9 \%$ (2/237) and 24 weeks in $0.9 \%(2 / 237)$. All patients were informed about the risk of possible complications and signed the written consent. The procedure was performed by two experienced operators with the use of 22 gauze needle and a needle-guide attached to the ultrasound probe, under the local anesthesia. Fetal viability was checked before and after the amniocentesis. It was performed under ultrasound guidance with continuous visualization of the needle. Approximately, $20 \mathrm{ml}$ of amniotic fluid was obtained. In cases of unfavorable placental location, on the front wall of the uterus or reduced amniotic fluid volume, the patients were informed about increased procedure risk [12]. To avoid the risk of $\mathrm{Rh}$ sensitization the anti-D prophylaxis was applied in $\mathrm{Rh}(-)$ negative patients. The results of karyotype analysis were available in about three weeks, due to the time necessary for growing the cell culture.

\section{RESULTS AND DISCUSSION}

The definitive chromosomal diagnosis can only be made from fetal cells which are available from the invasive tests: CVS, cordocentesis or amniocentesis.
CVS involves aspiration of placental tissue under ultrasound guidance and is usually performed between 11 and $13+6$ weeks [9]. There are two approaches to CVS: transabdominal and transcervical. Transcervical CVS has a higher incidence of spontaneous pregnancy loss, but it is a preferred method if the placenta is posterior or if the bowel enables a transabdominal approach. The main advantage of CVS is the early and definitive chromosomal analysis [13]. However, it is an invasive test that carries a high risk of pregnancy loss - from 0.6 to 4.6 percent (most frequently: miscarriage, infection, bleeding, limb defects) [14]. CVS performed before 10 weeks' gestation is associated with an increased risk of limb reduction defects from 1 to 2 percent [13]. Chromosome analysis is carried out either as a direct preparation or following brief culture (1 day) as well as after full culture ( 7 to 10 days).

During cordocentesis, which is a fairly difficult and technically challenging intervention, a small amount, usually $0.5-1 \mathrm{ml}$, of fetal blood is collected $[15,16]$. A sample of blood is obtained from the umbilical vein (close to the placenta) usually at 18-23 weeks of gestation under ultrasound guidance. The most common indications include suspected fetal anemia in association with rhesus disease, parvovirus B19 infection, or fetal hydrops. This procedure can also be applied for rapid karyotyping or molecular genetic diagnosis, depending on indication, especially, when an immediate result is needed, e.g. the presence of ultrasonographically detected anomalies or severe intrauterine growth restriction (IUGR). The result can be available in up to five days. The complication rate is estimated to be around $2 \%$ with the most common being: fetal bradycardia (usually short-lasting), bleeding, premature birth and fetal death.

An amniocentesis is one of the most frequently used invasive procedures with relatively low risk to both the mother and the fetus. It involves a transabdominal approach, with obtaining the amniotic fluid under ultrasound guidance [7]. The major disadvantage of amniocentesis is that the result is usually not available until the 18th week of gestation. Early amniocentesis, defined as amniocentesis performed between 9 and 14 weeks, is likely to be associated with a significantly higher risk of fetal loss than either CVS or amniocentesis performed after the 16th week of gestation $[17,18]$. The aim is to obtain fetal cells derived from skin, mucous membranes, amnion and umbilical cord for karyotyping or a DNA analysis. Chromosomal analysis usually requires prior cell culture, which on average takes two weeks. Then metaphase chromosomes are analyzed numerically and structurally. Uncultured amniotic fluid can be used to determine levels of AFP, which is present in increased concentrations in open neural tube or abdominal wall defects. In the presence of a raised AFP level, acetylcholinesterase is measured as a marker of neural tube defects.Unfortunately invasive methods can cause complications, even if the fetus is normal. The risk of complications following amniocentesis was lowered in the last decades by improvement of the technique, especially by the use of ultrasound scan [19]. But still the risk of pregnancy loss is about $0.5-1 \%$. Miscarriage, transient amniotic fluid leakage, fetal injury and intrauterine infection may occur $[7,12,20]$. It has to be emphasized that all forms of prenatal testing must be voluntary [21,22]. 
The most common indications for the procedure were: advanced maternal age $-86(36.2 \%$ of cases $)$, improper ultrasound scan -71 patients (29.8\%), improper biochemical screening tests -77 patients $(32.6 \%)$ history of a child with a chromosomal abnormality $-13(5.7 \%)$. The indications were similar to those presented by other authors $[5,7,8]$. The follow-up revealed one spontaneous abortion within 7 days after the procedure. Premature birth occurred in 14 cases: two of them had chromosomal abnormalities (Patau Syndrome and Down Syndrome). In other premature cases chromosome analysis detected no abnormalities. It is not easy to assess the rate of fetal loss following invasive procedures, because there is no consensus for how long monitoring of pregnancy should be performed for after amniocentesis. No neonatal deaths being the result of amniocentesis were noticed. In our study there were 3 cases of intrauterine fetal death at 3-5 weeks post-procedure, all fetuses had Turner Syndrome. Chromosomal abnormalities were detected in 33 patients which is $13.9 \%$ of all cases $(47 \mathrm{XX}+13,46 \mathrm{XX} 9 \mathrm{qht}, 45 \mathrm{X0}, 47 \mathrm{XY}+18$, 47XX+18, 47XY+13, 69XXY, 46XY,t(1;19)(p34.3;q13.3), $47 \mathrm{XX}+21 ， 46 \mathrm{XX}, \operatorname{der}(22) \mathrm{t}(18 ; 22)(\mathrm{q} 11.2 ; \mathrm{p} 11.2)$. Of these, trisomy 21 in 7 out of $33(20 \%)$, sex chromosomal abnormalities in $11(35 \%)$, triploid in $1(5 \%)$, and other chromosomal structural abnormalities in 13 out of $33(40 \%)$. In this group the main indications were: improper ultrasound scan ( 28 out of 33 cases) and the first trimester biochemical tests (16 out of 33 cases). In most cases there were at least two indications to perform amniocentesis. The chromosome results were normal in $85.1 \%(201 / 237)$ of cases. Pregnants with Down Syndrome fetuses were: 18, 41, 42 and 43 years old. The outcome of pregnancies was as follows: live births in $95.6 \%(226 / 237)$, intrauterine fetal deaths in $2.1 \%$ (5/237), miscarriages in $1 \%$ at 21 weeks ( 1 week after the amniocentesis), terminations in $1.4 \%$ (3/237, due to Edwards Syndrome, Patau and triploid). Amniocentesis followed by prenatal karyotyping is a reliable procedure, but like all tests, limited by factors, which can be of technical or biological nature. The probability of obtaining no fetal cellular material is less than $1 \%$ in experienced hands. In our study we also had one case of karyotyping not performed because of the lack of fetal cellular cells. A further limitation relates to the detection of a possible chromosomal mosaic, where two or more cell lines can be present. A mosaic can only be detected if chromosomally aberrant cells are present in the examined specimen.

\section{CONCLUSIONS}

The National Health Fund (NFZ) provides funding for only three medical centers performing complex screening and invasive procedures located in Lublin Voivodeship. This is highly insufficient since in the region there is a huge demand for this kind of service.

Educating both the patients and health care providers on the opportunities that the diagnostics of chromosomal defects give, should be the top priority.

During our research, we detected chromosomal abnormalities in 33 out of 237 patients, which is $13.9 \%$ out of total. The main indications were improper ultrasound scan and the first trimester biochemical, noninvasive screening tests. This is a proof that the mother's age and poor obstetrics history are huge risk factors and that modern, non-invasive procedures, such as first-trimester screening tests should be provided to every pregnant woman.

\section{REFERENCES}

1. Copel JA, Bahado-Singh RO. Prenatal screening for down's syndrome a search for the family's values. N Engl J Med. 1999;341(7):521-2.

2. Cleary-Goldman J, Morgan MA, Malone FD, et al. Screening for down syndrome: Practice patterns and knowledge of obstetricians and gynecologists. Obstet Gynecol. 2006;107:11-7.

3. Kuppermann M, Norton ME. Prenatal testing guidelines: Time for a new approach. Gynecol Obstet Invest. 2005;60:6-10.

4. Nicolaides KH, Sebire NJ, Snijders RJ. Down's syndrome screening with nuchal translucency. Lancet. 1997;349:438.

5. Larion S, Warsof SL, Romary L, et al. Association of combined firsttrimester screen and noninvasive prenatal testing on diagnostic procedures. Obstet Gynecol. 2014;123(6):1303-10.

6. Lau GW, Feldman DS, Morales CM, et al. First-trimester aneuploidy screening: is there a maternal age at which it loses effectiveness? J Reprod Med. 2014;59(9-10):443-7.

7. Schmidt W, Gabelmann J, Muller U, et al. Genetic amniocentesis: Technique and results in 1,000 first trimester amniocenteses. Geburtshilfe Frauenheilkd. 1980;40:761-8.

8. Weise W, Gabriel D. Report Of 8 years' experience in the prenatal diagnosis of genetic defects. Results. Zentralbl Gynakol. 1984;106:21629.

9. Zergollern L, Lasan R, Relja Z. The usefulness of the chorionic villi technic in prenatal diagnosis. Jugosl Ginekol Perinatol. 1989;29:50-4.

10. Zolotukhina TV, Kuznetsov MI, Kostiuk EV, et al. Detection of chromosomal abnormalities using cordocentesis. Genetika. 1991;27:1481-5.

11. Lo YM, Corbetta N, Chamberlain P, et al. Presence of fetal DNA in maternal plasma and serum. Lancet. 1997;350:485-7.

12. Lenke RR, Ashwood ER, Cyr DR, et al. Genetic amniocentesis: Significance of intraamniotic bleeding and placental location. Obstet Gynecol. 1985;65:798-801.

13. Cunningham FG, Leveno KL, Bloom SL, et al. Williams Obstetrics, NY: Mcgraw-Hill; 2005. p. 328-30.

14. Jenkins TM, Wapner RJ, Khalek N. Prenatal diagnosis of congenital disorders. Maternal-Fetal Medicine: Principles and Practice; 2004. p. 263-9.

15. Nicolaides KH. Cordocentesis. Clin Obstet Gynecol. 1988;31:123-35.

16. Nicolaides KH, Rodeck CH. Fetal blood sampling. Baillieres Clin Obstet Gynaecol. 1987;1:623-48.

17. Canadian Early and Mid-Trimester Amniocentesis Trial (CEMAT) Group. Randomised trial to assess safety and fetal outcome of early and midtrimester amniocentesis. Lancet. 1998;351:242-7.

18. Nicolaides KH, Brizot M De L, Patel F, Snijders R. Comparison of chorionic villus sampling and amniocentesis for fetal karyotyping at 10-13 weeks' gestation. Lancet. 1994;344:435-9.

19. Cruz-Lemini M, Parra-Saavedra M, Borobio V, et al. How to perform an amniocentesis. Ultrasound Obstet Gynecol. 2014;44(6):727-31.

20. Antsaklis A, Papantoniou N, Xygakis A, et al. Genetic amniocentesis in women 20-34 years old: associated risks. Prenat Diagn. 2000;20:24750 .

21. Comas C, Echevarria M, Rodríguez I, et al. Prenatal invasive testing: a 13-year single institution experience. J Matern Fetal Neonatal Med. 2014;27(12):1209-12.

22. Nicolaides KH, Chervenak FA, Mccullough LB, et al. Evidence-based obstetric ethics and informed decision-making by pregnant women about invasive diagnosis after first-trimester assessment of risk for trisomy 21. Am J Obste Gynecol. 2005;193:322-6.

\section{Corresponding author}

Arkadiusz Krzyżanowski

16 Staszica Str., 20-081 Lublin

Tel.: 815326612 ,

E-mail:a_r_krzyzanowski@o2.pl 